\title{
Optimization of SUAV's propeller in a hover
}

\author{
Jakub Hnidka ${ }^{1, *}$, Dalibor Rozehnal ${ }^{1}$ and Karel Maňas ${ }^{1}$ \\ ${ }^{1}$ University of Defence in Brno, Kounicova 65, 60200 Brno, Czech Republic
}

\begin{abstract}
Small unmanned aerial vehicles (SUAVs) have found a widespread application in past decades. However, as the criticality of the missions for which they can be used increases, the demand for improvement of their efficiency increases as well. The paper focuses on a propeller driven SUAVs of a multirotor type, equipped with an electric motor, battery and propeller. The paper presents a simplified method of calculation of the SUAV maximal endurance, if the characteristics of all components of the propulsion system are known. To improve the overall efficiency of the propulsion system of an SUAV, the correct combination of all propulsion system components is critical. However, the largest impact on the maximal endurance is, arguably, caused by the propeller. The paper proposes a simple method of optimizing the propeller characteristics for hover and compares the proposed propeller design with conventional and commercially available propellers.
\end{abstract}

\section{Introduction}

The field of application of SUAVs in the past decades has been growing and the demand for their better flight performance, especially for challenging applications such as meteorological measurements or surveillance and scanning operations to e.g. quickly map a large area to determine a presence of life-threatening substances, has been growing as well. SUAVs, while reliable, light and quick to deploy even in swarm formations can often meet a number of these requirements. Arguably, the most challenging parameter to tune is their maximal endurance. The root of this problem lies in the propulsion system itself, which often consists from a battery, electric motor and propeller(s) [1]. The high efficiency of electric motors cannot offset the lack of energy available on board even with the cuttingedge batteries. The entire propulsion system has to be carefully tuned to improve its overall efficiency. Correct combination of the battery, motor and propeller has time and time proven to be critical to achieve this goal.

The paper presents a quick overview of the characteristics of all components of the propulsion system and a simple algorithm, which combines all characteristics to obtain the maximal endurance. It also presents a rapid blade element method of calculating the propeller performance characteristics. With evolution algorithms, this method could be used to obtain new, non-conventional geometries, which could further increase the efficiency of the propulsion system of the SUAV.

*Corresponding author: jakub.hnidka@unob.cz 


\section{SUAV powertrain and maximal endurance}

The propulsion system of the SUAV consists most commonly from a battery, BLDC electric motor and a fixed-pitch propeller. Due to their high energy density, typically, LiIon batteries are used. In a hover, the propeller needs to produce thrust equal to the weight or a portion of the weight of the SUAV. Fixed-pitch propeller can achieve this thrust only at a single setting of the propeller revolutions, e.g. at a certain throttle setting. However, as the battery is discharging, the voltage in the entire system drops and at the same throttle setting, the revolutions of the brushless DC (BLDC) motor and the propeller would drop as well. The flight control system has to increase the throttle setting to keep the SUAV in a hover. At certain discharge level, there would no longer be possible to further increase the throttle setting and the SUAV would start falling to the ground.

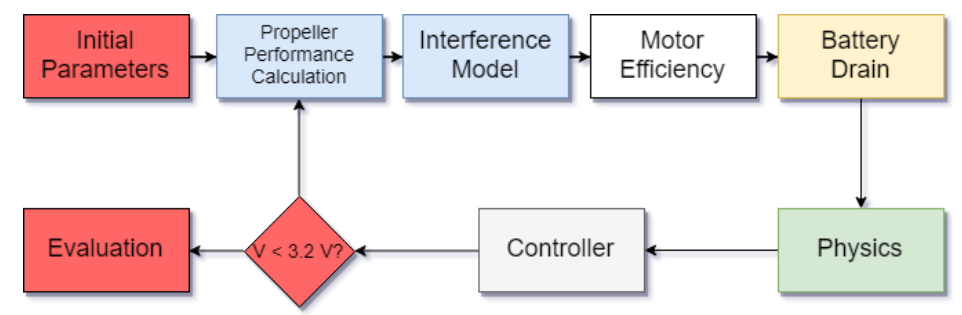

Fig. 1. Algorithm to calculate the maximal endurance of an SUAV in a hover [1].

The algorithm, which implements this thought-process can be shown in the Fig. 1. The interference model depends on the mechanical installation of the propeller on the fuselage of the SUAV. The physics part contains equations of motions to change the attitude of the SUAV in the environment, if needed. The controller module contains a simple PID regulator to increase the revolutions of the electric motor. The calculation is repeated with a selected time step until cut-off voltage level is achieved and the battery is considered fully discharged. Propeller Performance Calculation, Motor Efficiency and Battery Drain contain characteristics of propeller, BLDC motor and battery.

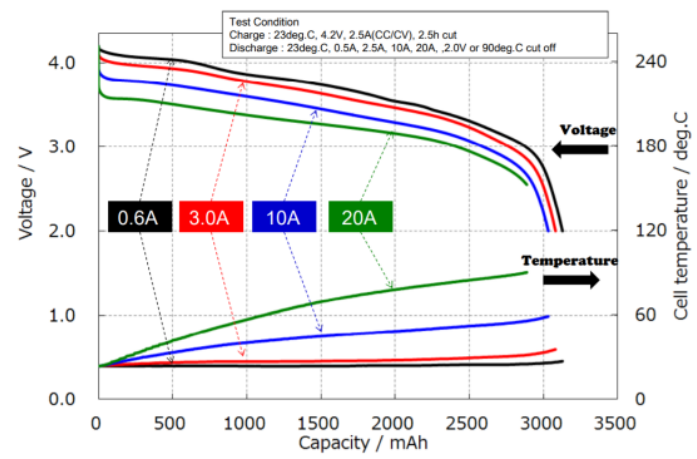

Fig. 2. Discharge load characteristics of Sony VTC-6 [2]. 
The battery discharge characteristics of a commercially available Sony US18650VTC6 are shown in Fig. 2. The characteristic shown is typical for Li-Ion battery. At first, the voltage drops significantly from ca $4.2 \mathrm{~V}$, then the rate of discharge levels until it drops significantly, and battery falls into deep-discharge area. As the voltage in the system drops, the current increases, which further effects the characteristics. To obtain the largest possible flight endurance, it is critical that the cut-off voltage does NOT crosses the characteristic in its levelled part. Any increase of the current in the system can have a devastating impact on the amount of energy the system is capable of actually draining from the battery. The discharge load characteristics is impacted also by ambient temperature and cycle life performance.

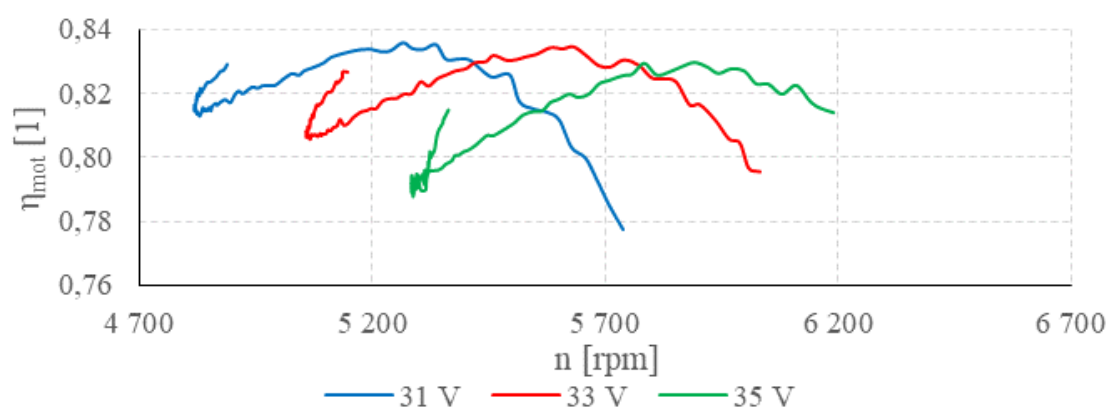

Fig. 3. Measured efficiency of a BLDC motor [1].

The measured efficiency of the BLDC motor is shown in Fig. 3. The measurement was started with the cold motor. As the motor heated up, the internal resistance was increased, which caused it to drop some revolutions and it decreased its efficiency.

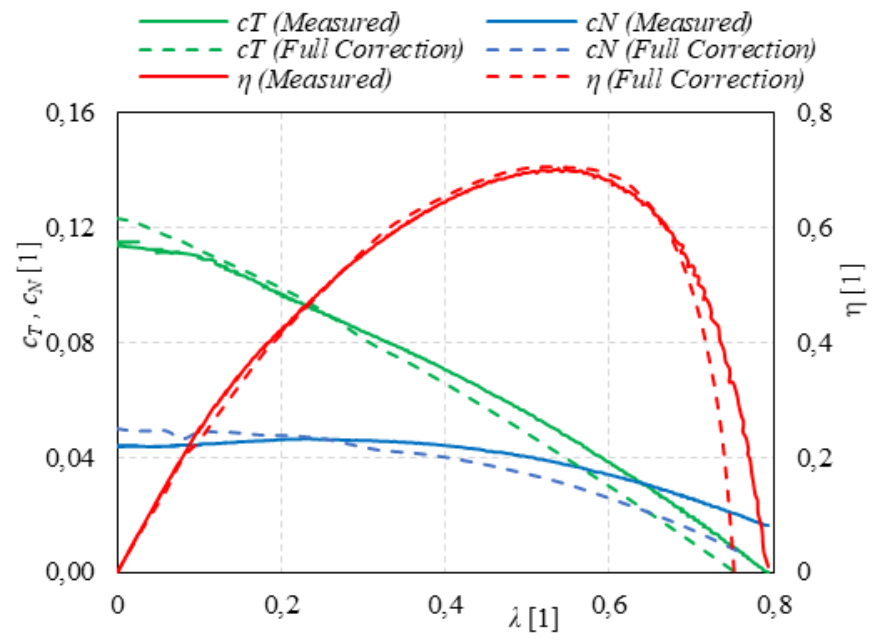

Fig. 4. Comparison of measured and calculated performance characteristics [3].

The propeller performance characteristics can be both measured and calculated. Measured and calculated performance characteristics of Bambula $18 \times 8$ are shown in the Fig. 4 . The 
propeller performance characteristics plots coefficient of thrust $c_{T}$, coefficient of power $c_{N}$ and efficiency $\eta$ against the advance ratio $\lambda$. Efficiency and advance ratio are defined as

$$
\begin{aligned}
& \eta=\frac{c_{T}}{c_{N}} \lambda \\
& \lambda=\frac{V}{n D}
\end{aligned}
$$

The forward velocity of the propeller is noted as $V$.

\section{Calculation of propeller performance characteristics}

Measurement of the propeller performance characteristics, while highly precise is also costly and time consuming, and requires wind tunnel. To overcome this problem, a number of methods of calculating the propeller performance characteristics was introduced during the past century. Blade element methods $[4,5]$, vortex theory $[6,7]$, generalized dynamic wake model [8], or CFD [9]. Blade element methods are often used to calculate the performance of the wind turbines as well [10]. This paper presents an algorithm based on the Joukowsky blade element model, closely described in [3]. The validation of this model along with an introduction of different correction methods was performed in $[3,11]$.

At its core, the method divides the blade of the propeller into finite number of blade sections. On these sections, a 2D uniform flow is assumed and the 3D problem of the flow over propeller blade is simplified into much simpler problem of calculation of an aerodynamic force acting upon an aerofoil. These forces are then integrated along the propeller blade and a thrust of the propeller is found. This method provides a rapid and fairly precise (see Fig. 4) solution to the problem of calculation of propeller performance characteristics.

\section{Implementation of evolution algorithms to improve propeller efficiency}

The propeller arguably contributes to the overall efficiency of the propulsion system the most. For purposes of design optimization of the propeller, the ratio between the coefficient of thrust $c_{T}$ and coefficient of power $c_{N}$ was defined as follows

$$
\frac{c_{T}}{c_{N}}=\frac{T}{N} \frac{\rho n^{3} D^{5}}{\rho n^{2} D^{4}}
$$

The propeller has the diameter $D$, spins at revolutions $n$, the density of the air is noted as $\rho$ and the propeller produces thrust $T$ and consumes power $N$. Improving this ratio for a general propeller can be challenging, because the propeller typically does not operate at a single point, but has to perform well in a broad range of the advance ratio $\lambda$. To simplify this problem, a hover $(\lambda=0)$ was assumed.

This optimization problem applied to a Joukowsky blade element model can be expressed as follows

$$
\left(\frac{c_{T}}{c_{N}}\right)_{\max }=\frac{1}{\pi}\left(\frac{\int_{0}^{1} \bar{\Gamma}\left(\overline{U_{1}}-\kappa \overline{V_{1}}\right) \mathrm{d} \bar{r}}{\int_{0}^{1} \bar{\Gamma}\left(\overline{V_{1}}+\kappa \overline{U_{1}}\right) \bar{r} \mathrm{~d} \bar{r}}\right)_{\max }
$$


The $\bar{r}$ is dimensionless local radius referenced to radius of the blade, $\kappa$ is local lift to drag ratio and $\bar{\Gamma}$ is a dimensionless local circulation, expressed as

$$
\bar{\Gamma}=\frac{\Gamma}{4 \pi \Omega R^{2}}
$$

The $\Omega$ is angular velocity of the propeller, $\Gamma$ is a local value of circulation and $R$ is radius of the propeller. Velocities $\overline{U_{1}}$ and $\overline{V_{1}}$ can be obtained as

$$
\begin{gathered}
\overline{U_{1}}=\bar{r}-\frac{\bar{\Gamma}}{\bar{r}} \\
\overline{V_{1}}=\sqrt{\bar{\Gamma}(1-\bar{\Gamma})}
\end{gathered}
$$

The solution heavily depends on the aerofoil polar of each blade section and its lift to drag ratio, which is not only geometry dependant, but it is also impacted by the value of the local Reynolds number. To rapidly obtain required aerofoil polars, XFOIL by Drela was used.

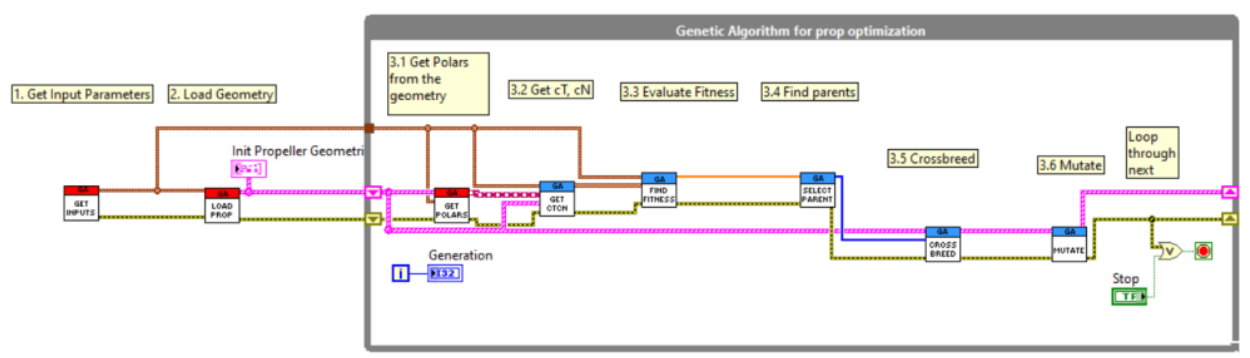

Fig. 5. Implementation of evolution algorithm in LabVIEW.

The algorithm of the propeller evolution is shown in the Fig. 5. At first, 10 initial geometries are selected as a poll from commercially available propeller geometries. Then, a blade element model is applied to obtain results of the propeller performance characteristics of each propeller. A fitness function is used to find three propellers with minimal values of power coefficient $c_{N}$, while still producing required thrust. In the next step, these three parents are used for crossbreeding, where another six siblings are created. Three parents are always carried to next generation to keep the best solutions. The tenth sibling has the same geometry as the best parent, but it is subjected to mutation. During mutation, some of the parameters are varied to produce slightly different geometry of the propeller. In next step, the propellers are again evaluated, by obtaining the propeller performance characteristics and getting the values of the fitness function. The fitness function scores the solutions based on following equation

$$
F=w_{1} c_{T}-w_{2} \frac{c_{T}}{c_{N}}
$$

To assure convergence to a reasonable solution, a required thrust has to be selected before the calculation. Closeness to the required thrust is awarded by a weight $w_{l}$. Secondly, the efficiency of the solution is evaluated as well. 


\section{Comparison of proposed designs}

The results of this optimization are shown in the Fig. 6. It is important to mention that evolution algorithms in no way ensure the convergence to the best solution and the result may vary with each run of the algorithm. However, due to the significant increase in the computational power in the past decade, it is possible to use the artificial intelligence to tune the propeller design - even if it is only in a limited scope.

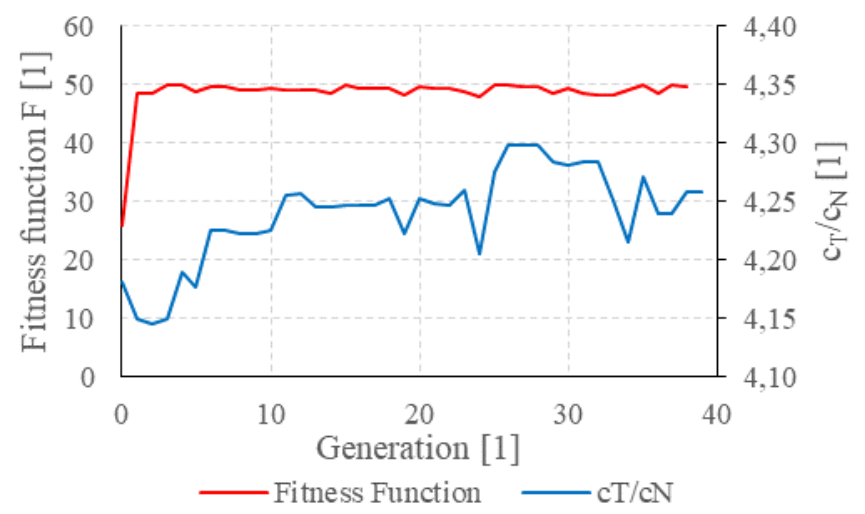

Fig. 6. Best parent after 40 generations.

The optimization brought only a minor improvement over the original design. However, as seen in Fig. 7, the ability to choose appropriate propeller designs has drastic impact on future designs. This rapid choice of propeller from large database of propeller designs, affected by initial conditions of the calculation is in itself a useful feature of the program.

In the initial generation, there was a propeller with $c_{T} / c_{N}$ ratio equal to 6 , which is larger than any other proposed design. However, this propeller failed to produce required thrust at given settings, which is why it was rejected.

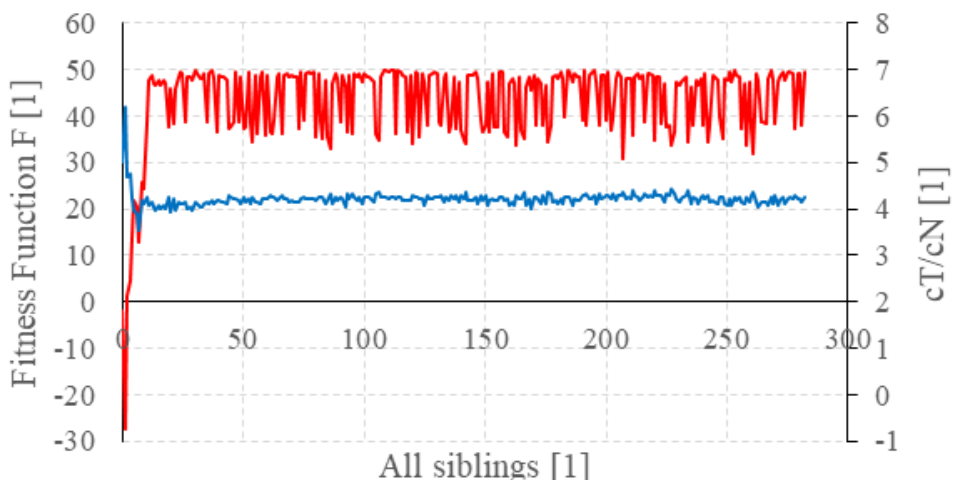

- Fitness function $-\mathrm{cT} / \mathrm{cN}$

Fig. 7. Change of the fitness factor of the best parent of each generation. 
The problem converges to a solution with a constant local circulation distribution along the blade of the propeller, which is to be expected. A calculated propeller can be seen in Fig. 8. The pitch angle of each blade section roughly corresponds to an angle of attack of best range regime (lift-to-drag ratio to the power of 1.5). Chord characteristics is dictated by a distribution of a local circulation.

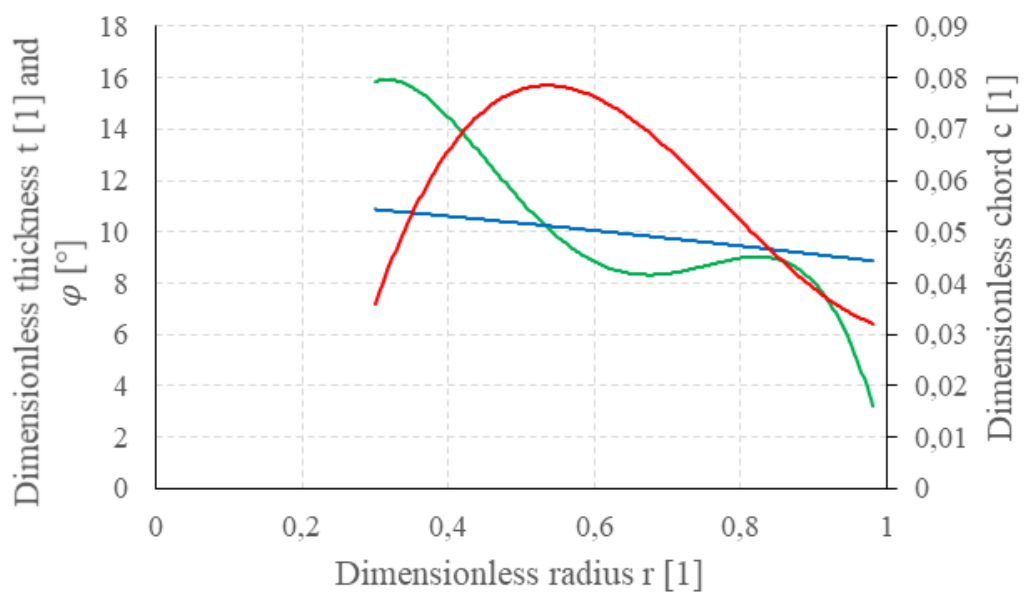

Fig. 8. Geometry of a calculated propeller.

\section{Conclusion}

Paper presents a simple method of calculating the maximum flight endurance of the SUAV and identifies a propeller as a main source of the inefficiency of the propulsion system. It also presents a rapid blade element model of calculation of the propeller performance characteristics. To improve the efficiency of the propeller, expressed as a ratio between the coefficient of thrust and power, evolution algorithm was proposed. The algorithm coupled with XFOIL to calculate the aerofoil polar for each blade section proved to be able to produce more efficient designs than commercially available propellers. It also proved to be highly efficient at choosing from commercially available designs the most suited propeller. However, the algorithm is tuned to optimize the propeller only for a single operating point - the paper presents results for hover, but this implementation produces results for non-zero values of a forward velocity as well. Future development will focus on widening the optimization for more than one operating point.

This research was sponsored by TECHNOFIBER.

\section{References}

1. J. Hnidka, D. Rozehnal, Calculation of the maximum endurance of a small unmanned aerial vehicle in a hover, IOP Conference Series: Materials Science and Engineering, 664, 1-9 (2019)

2. SONY, Lithium Ion Rechargeable Battery Technical Information, 1-10 (2015) 
3. J. Hnidka, D. Rozehnal, V. B. Nguyen, Evaluation of BEM Calculation Method used for Low-Reynolds Propellers, ICMT 2019, 1-5 (2019)

4. V.L. Okulov, J. N. Sørensen, D. H. Wood, The rotor theories by Professor Joukowsky: Vortex theories, Progress in Aerospace Theories, 73, 19-46 (2018)

5. M. C. Schneider, J. Nitzsche, H. Hennings, Accurate load prediction by BEM with airfoil data from 3D RANS simulations, Journal of Physics: Conference Series, (2016)

6. F. Panayotov, I. Dobrev, F. Massouh, F. Todorov, Numerical model for rapid computation of the flow-field of a rotor in hover, ICMT 2017, 570-577 (2017)

7. B. A. Moffitt, T. H. Bradley, D. E. Parekh and D. Mavris, Validation of Vortex Propeller Theory for UAV Design with Uncertainty Analysis, (2008)

8. S. Makinen, Applying dynamic wake models to large swirl velocities for optimal propellers (2005)

9. A. Kusyumov, S. Kusyumov, S. Mikhailov, E. Romanova, K. Phayzullin and E. a. B. G. Lopatin, Main rotor-body action for virtual blades model, Experimental Fluid Mechanics, (2017)

10. L. A.Viterna, R. D. Corrigan, Fixed pitch rotor performance of large horizontal axis wind turbines, Energy Production and Conversion, 69-85 (1982)

11. R. MacNeill D. Verstraete, Blade element momentum theory extended to model low Reynolds number propeller performance, The Aeronautical J., 121, 835-57 (2017) 